\title{
Pazopanib and Statin-Induced Rhabdomyolysis
}

\author{
Jennifer M. Logue ${ }^{a, b} \quad$ Bahram Kiani $^{a, c}$ Rhonda L. Bitting ${ }^{a, b, d}$ \\ ${ }^{a}$ Wake Forest School of Medicine, Winston-Salem, NC, USA; ${ }^{b}$ Department of Internal \\ Medicine, Wake Forest School of Medicine, Winston-Salem, NC, USA; ${ }^{C}$ Department of \\ Radiology, Wake Forest School of Medicine, Winston-Salem, NC, USA; ${ }^{d}$ Wake Forest \\ Baptist Comprehensive Cancer Center, Wake Forest Baptist Medical Center, Winston- \\ Salem, NC, USA
}

\section{Keywords}

Pazopanib - Rosuvastatin - Rhabdomyolysis - VEGF inhibitor · Statin · Hyperlipidemia $\cdot$ Renal cell carcinoma $\cdot$ Cytochrome p450

\begin{abstract}
Background: The VEGF inhibitor pazopanib is a widely used first-line therapy for the treatment of advanced renal cell carcinoma. Potential drug-drug interactions and toxicities may be underrecognized. Case Presentation: A 73-year-old woman with metastatic renal cell carcinoma on treatment with pazopanib presented with progressive inability to ambulate. The initial concern was for metastasis to the spine. However, MRI of the spine revealed diffuse muscle edema with no metastatic deposits or lytic lesions. Upon further evaluation, creatine kinase was significantly elevated and the diagnosis of rhabdomyolysis was made. With aggressive hydration and discontinuation of both pazopanib and rosuvastatin, the patient made a full recovery. Conclusion: This case of drug-induced rhabdomyolysis demonstrates an unexpected toxicity resulting from concomitant pazopanib and rosuvastatin therapy. This combination is predicted to be safe due to different, nonoverlapping effects on the cytochrome p450 enzymes. Discontinuation of statin therapy in patients with metastatic cancer should be considered when the risk of cancer-related death exceeds the risk of cardiovascular-related death.




\section{Background}

The VEGF tyrosine kinase inhibitor pazopanib is a widely used first-line therapy for the treatment of advanced renal cell carcinoma, on the basis of large studies which demonstrated efficacy and safety $[1,2]$. With the increased use of any drug, rare side effects are expected to manifest. Here, we describe a patient with statin-induced rhabdomyolysis, most likely due to inhibition of the cytochrome $\mathrm{p} 450$ system by pazopanib.

\section{Case Presentation}

A 73-year-old woman presented with gross hematuria, and a large right renal mass was discovered. She underwent right radical nephrectomy, and pathology revealed clear cell renal cell carcinoma. Postoperative imaging showed nodularity in the nephrectomy bed, multiple hepatic lesions, bilateral pulmonary nodules, and mediastinal adenopathy, giving concern for residual metastatic disease. She started pazopanib $600 \mathrm{mg}$ daily, initially $25 \%$ dose-reduced due to frailty. Due to hypertensive urgency, the dose was further decreased to $400 \mathrm{mg}$ daily during the first month of treatment. Other early treatment side effects included elevated TSH with normal $\mathrm{T}_{3}$ and $\mathrm{T}_{4}$ and grade 1 thrombocytopenia. Follow-up imaging after 3 months of treatment showed a partial response, with tumor regression noted in all affected areas.

After 6 months of treatment with pazopanib $400 \mathrm{mg}$, she presented to the clinic for routine follow-up and reported 9 days of progressive bilateral lower extremity weakness, ultimately requiring wheelchair use. She also reported worsening of her chronic lower back pain. She had intentionally decreased her fluid intake in order to decrease her urine output, as it had become too difficult to get to the bathroom at home. Physical examination revealed mildly decreased but symmetrical lower extremity strength and diffuse tenderness of the lumbar and sacral spine and paraspinal region. Initial laboratory findings were notable for a BUN level of $51 \mathrm{mg} / \mathrm{dL}$ and creatinine of $2.82 \mathrm{mg} / \mathrm{dL}$, up from her baseline of $1.5 \mathrm{mg} / \mathrm{dL}$. She also had a new transaminitis with AST of $172 \mathrm{IU} / \mathrm{L}$ (normal 5-40 IU/L) and ALT of $158 \mathrm{IU} / \mathrm{L}$ (normal 5-50 IU/L).

She was given intravenous fluids and admitted to the hospital for further workup. MRI of the lumbosacral spine revealed diffuse paraspinal muscle edema from L2 to the sacrum, without evidence of osseous metastasis to the spine (Fig. 1). Further evaluation revealed creatine kinase of 5,719 IU/L (normal 50-160 IU/L) and a fractional excretion of sodium of $2.42 \%$, consistent with intrinsic renal injury caused by rhabdomyolysis. On review of her home medications, she had been taking rosuvastatin $40 \mathrm{mg}$ daily for several years for hyperlipidemia. Her statin was stopped due to concern for statin-induced rhabdomyolysis. Pazopanib was also stopped. With continued fluid resuscitation, her creatine kinase normalized and her renal and hepatic function recovered to baseline. After several weeks of physical therapy, she returned to her baseline physical functioning and performance status.

\section{Discussion}

The prescribing information for pazopanib carries a warning with regard to the severe and fatal hepatotoxicity observed in clinical trials, though there are no absolute contraindications listed. Per Novartis, the most common adverse reactions when treating for advanced 
renal cell carcinoma are diarrhea, hypertension, hair color changes, nausea, anorexia, and vomiting [3]. In vitro studies suggest that pazopanib undergoes oxidative metabolism in human liver microsomes, primarily via CYP3A4, with minor contributions from CYP1A2 and CYP2C8. Results from drug-drug interaction trials suggest that pazopanib is a weak inhibitor of CYP3A4, CYP2C8, and CYP2D6 in vivo, and concomitant use of agents with narrow therapeutic windows that are metabolized by these enzymes is not recommended. Pazopanib has no known effect on CYP2C9. Concomitant use of pazopanib and simvastatin has been shown to increase the risk of ALT elevations, and providers are instructed to use this combination with caution and close monitoring. There is presently insufficient data, however, to assess the risk of alternative statins given in combination with pazopanib [3].

Use of statins in the presence of known drug interactions has been extensively studied in the treatment of HIV due to the high prevalence of dyslipidemia in patients receiving highly active antiretroviral therapy (HAART) and the CYP3A4 inhibition commonly caused by HAART. As a result, nondrug therapies such as dietary and exercise interventions are first recommended and given a thorough trial. When there is an urgent need to intervene in the case of hyperlipidemia, pravastatin is recommended because it is predominantly metabolized outside of the liver. Simvastatin and lovastatin have major interactions with CYP3A4 and are generally avoided. Atorvastatin also interacts with CYP3A4 but to a lesser degree [4]. Rosuvastatin, in contrast, is metabolized predominantly through CYP2C9 [5]. Because current guidelines recommend high-intensity statin therapy for both primary and secondary prevention of atherosclerotic cardiovascular disease, side effects such as rhabdomyolysis are seen more commonly. The most important consideration to reduce such side effects is to limit concomitant use of CYP3A4 inhibitors or inducers [5]. In the case reported here, statininduced rhabdomyolysis occurred with concomitant pazopanib and rosuvastatin use, despite the fact that rosuvastatin is predominantly metabolized by CYP2C9.

\section{Conclusion}

This case of statin-induced rhabdomyolysis demonstrates a severe effect of cytochrome P450 inhibition due to pazopanib. Though the drug-drug interaction studies suggest that pazopanib is a weak inhibitor only of CYP3A4, CYP2C8, and CYP2D6, this patient's experience would argue that the inhibition may be more potent than suspected and that CYP2C9 may also be affected. Discontinuation of statin therapy for patients with metastatic cancer should be considered when the risk of cancer-related death exceeds the risk of cardiovascular-related death.

\section{Statement of Ethics}

The authors have no ethical conflicts to declare.

\section{Disclosure Statement}

The authors have no relevant disclosures or conflicts of interest. JL and RB analyzed and interpreted the patient data in the context of the literature. BK provided the radiologic interpretation. All authors read and approved the final manuscript. 


\section{Case Reports in Oncology}

\section{References}

-1 Sternberg CN, Hawkins RE, Wagstaff J, et al: A randomised, double-blind phase III study of pazopanib in patients with advanced and/or metastatic renal cell carcinoma: final overall survival results and safety update. Eur J Cancer 2013;49:1287-1296.

-2 Motzer RJ, Hutson TE, Cella D, et al: Pazopanib versus sunitinib in metastatic renal-cell carcinoma. N Engl J Med 2013;369:722-731.

3 Novartis Pharmaceuticals Corporation: Votrient (pazopanib) tablets: US prescribing information. 2017. https://www.pharma.us.novartis.com (accessed July 5, 2017).

4 Mayer KH, Dube MP, Sprecher D, et al: Preliminary guidelines for the evaluation and management of dyslipidemia in adults infected with HIV and receiving antiretroviral therapy: recommendations of the Adult AIDS Clinical Trial Group Cardiovascular Disease Focus Group. Clin Infect Dis 2000;31:12161224.

5 Benes LB, Bassi NS, Davidson MH: The risk of hepatotoxicity, new onset diabetes and rhabdomyolysis in the era of high-intensity statin therapy: does statin type matter? Prog Cardiovasc Dis 2016;59:145-152.

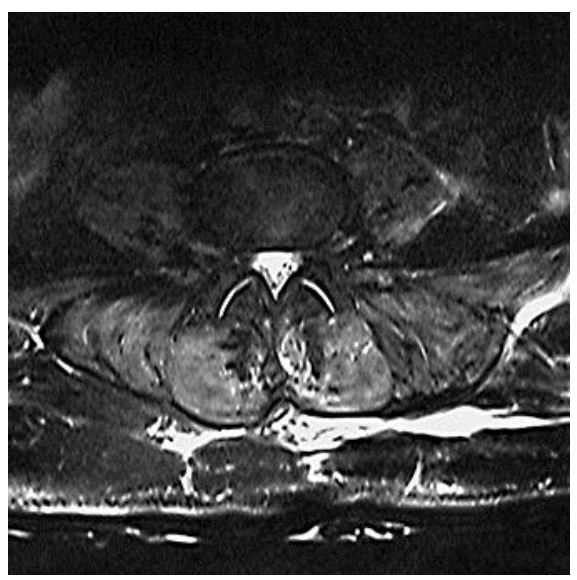

Fig. 1. MRI of the lumbosacral spine showing diffuse paraspinal muscle edema from L2 to the sacrum, without evidence of osseous metastasis to the spine. 